07

\title{
Статистические закономерности формирования магистральной трещины в структурно-неоднородном материале при различных условиях деформирования
}

\author{
(C) Е.Е. Дамаскинская ${ }^{1}$, В.Л. Гиляров ${ }^{1}$, И.А. Пантелеев ${ }^{2}$, Д.Р. Гафуурова ${ }^{3}$, Д.И. Фролов ${ }^{1}$ \\ ${ }^{1}$ Физико-технический институт им. А.Ф. Иофрее РАН, \\ Санкт-Петербург, Россия \\ ${ }^{2}$ Институт механики сплошных сред УрО РАН, \\ Пермь, Россия \\ ${ }^{3}$ Московский государственный университет им. М.В. Ломоносова, \\ Москва, Россия \\ E-mail: Kat.Dama@mail.ioffe.ru
}

(Поступила в Редакцию 26 марта 2018 г.)

\begin{abstract}
Проведено исследование особенностей формирования магистральной трещины в образцах гранита Westerly при квазистатическом одноосном сжатии без бокового подпора по данным акустической эмиссии (AE) и рентгеновской компьютерной микротомографии (СТ). Проведен мультифрактальный анализ пауз между событиями акустической эмиссии и анализ функционального вида распределений сигналов АЕ по энергии. На основании результатов компьютерной томографии установлено, что дефекты образуются только в области будущей магистральной трещины, т.е. стадии дисперсного накопления дефектов во всем объеме образца не выявлено. Обнаружены две стадии формирования магистральной трещины, первая характеризуется экспоненциальным распределением сигналов АЕ по энергии, вторая - степенным законом распределения сигналов АЕ по энергиям. Результаты мультифрактального анализа пауз между соседними сигналами $\mathrm{AE}$ демонстрируют переход от мультифрактальной динамики акустической эмиссии к монофрактальной при приближении к моменту разрушения.
\end{abstract}

Работа выполнена при финансовой поддержке Российского фонда фундаментальных исследований (гранты № 16-05-00237, 17-05-00720).

DOI: 10.21883/FTT.2018.09.46396.078

\section{1. Введение}

Многочисленные эксперименты по деформированию хрупких структурно-неоднородных материалов как природного происхождения, так и искусственно созданных показывают [1-4], что в условиях одноосного квазистатического сжатия с боковым подпором накопление дефектов происходит в несколько стадий. В начале нагружения дефекты образуются случайным образом во всем объеме. По мере деформирования происходит переход к стадии локализации дефектообразования в области, где в дальнейшем образуется макротрещина. В случае квазистатического одноосного сжатия таких материалов с боковой поверхностью, свободной от напряжений, стадия дисперсного накопления повреждений практически отсутствует [5]. Однако накопление повреждений и формирование магистральной трещины представляет собой нестационарный процесс, имеющий свои характерные нелинейные закономерности.

Данная работа посвящена исследованию статистических закономерностей формирования магистральной трещины при деформировании образцов гранита Westerly без бокового подпора для двух нетипичных условий нагружения: квазистатического одноосного сжатия с явно выраженной сдвиговой компонентой и ква- зистатического одноосного сжатия со снятием нагрузки незадолго до макроразрушения образца. Отличительной особенностью исследований является использование двух методов неразрушающего контроля: акустической эмиссии и рентгеновской микротомографии.

\section{2. Методика эксперимента}

Для исследования пространственного распределения дефектов и закономерностей их накопления при одноосном квазистатическом сжатии образцов гранита Westerly был выбран метод рентгеновской компьютерной микротомографии. Преимуществом данного метода является возможность получения данных о пространственно-временно́й картине развития дефектов (микротрещин) в объеме деформируемого материала и ее связи со структурными элементами материала, с сохранением целостности исследуемого объема.

Особенностью метода рентгеновской компьютерной микротомографии является то, что пространственное разрешение коррелирует с толщиной образца [6].

Поскольку целью экспериментов является исследование закономерностей образования и накопления дефектов в объеме образца горной породы, размеры образца должны быть достаточны для обеспечения возможности 
Минеральный состав гранита Westerly

\begin{tabular}{c|c|c|c|c|c}
\hline \multirow{2}{*}{ Минерал } & Кварц & Плагиоклаз & Полевой шпат & Биотит & Мусковит \\
\cline { 2 - 6 } & $28 \%$ & $33 \%$ & $33 \%$ & $3.5 \%$ & $1.9 \%$ \\
\hline Плотность & $2600-2650 \mathrm{~kg} / \mathrm{m}^{3}$ & $2620-2760 \mathrm{~kg} / \mathrm{m}^{3}$ & $2560 \mathrm{~kg} / \mathrm{m}^{3}$ & $2800-3400 \mathrm{~kg} / \mathrm{m}^{3}$ & $2760-3100 \mathrm{~kg} / \mathrm{m}^{3}$
\end{tabular}

деформирования и получения достоверных результатов локации сигналов АЕ. В результате была определена форма и размеры образцов, которые оптимальны как для механических испытаний, так и для томографии - это цилиндры диаметром $10 \mathrm{~mm}$ и высотой $20 \mathrm{~mm}$. Эксперименты проводились на образцах гранита Westerly [7], в котором средний размер зерна составляет $0.075 \mathrm{~mm}$ [8]. Минеральный состав приведен в таблице.

Пространственное разрешение томографических изображений при такой геометрии образца составило $\sim 3 \mu \mathrm{m}$. С учетом физических принципов томографии, конструктивных особенностей рентгеновской трубки и камеры томографа достигнутое разрешение является максимально возможным для образцов данного размера [6].

Исследование дефектной структуры образцов горных пород методом рентгеновской микротомографии до и после механических испытаний проводилось с помощью томографа ScyScan 1172 (Bruker, Belgium).

Томографическая съемка всей серии образцов, проведенная до начала механических испытаний, показала, что в исходных образцах отсутствуют дефекты типа трещин и пор с размерами более $3 \mu \mathrm{m}$. Это важный результат, поскольку дефекты, существующие до начала деформирования, могут приводить к концентрации напряжений (к повышенным по сравнению со средними локальным напряжениям), следовательно, предопределять место зарождения магистральной трещины. Поэтому для уточнения данного результата были проведены дополнительные исследования. Несколько образцов из серии были использованы для изготовления шлифов. Исследования были проведены сотрудниками Института вулканологии и сейсмологии ДВО РАН с помощью электронного микроскопа SEM Vega 3 Tescan и поляризационного микроскопа Nikon Eclipse LV 100 pol. Анализ изображений в отраженных электронах и фотографий шлифов не выявил дефектов типа трещин.

Механические испытания образцов гранита проводились на электромеханической испытательной машине AGX-Plus (Shimadzu, Japan). Для регистрации акустической эмиссии была использована система Amsy-5 (Vallen, Germany). Два широкополосных пьезопреобразователя акустической эмиссии AE105A с полосой $450-1150 \mathrm{kHz}$ крепились к торцам образца, что позволило осуществить линейную локацию источников сигналов АЕ. В процессе эксперимента формировалась база данных, в которой записаны параметры отдельных сигналов $\mathrm{AE}$ - время излучения, координата источника и энергия. Точность определения координат источников сигналов $\mathrm{AE} \sim 2 \mathrm{~mm}$.

Для исследования статистических закономерностей формирования магистральной трещины каждый образец подвергался одноосному квазистатическому сжатию с постоянной скоростью перемещения пуансонов $10 \mu \mathrm{m} / \mathrm{min}$. Кривые изменения напряжения в процессе деформирования приведены на рис. 1 При лавинообразном нарастании активности акустической эмиссии (более ста импульсов АЕ в секунду) процесс деформирования останавливался, образец разгружался и проводилась его томографическая съемка. Использованный критерий остановки процесса деформирования в совокупности с низкой скоростью деформирования позволил с одной

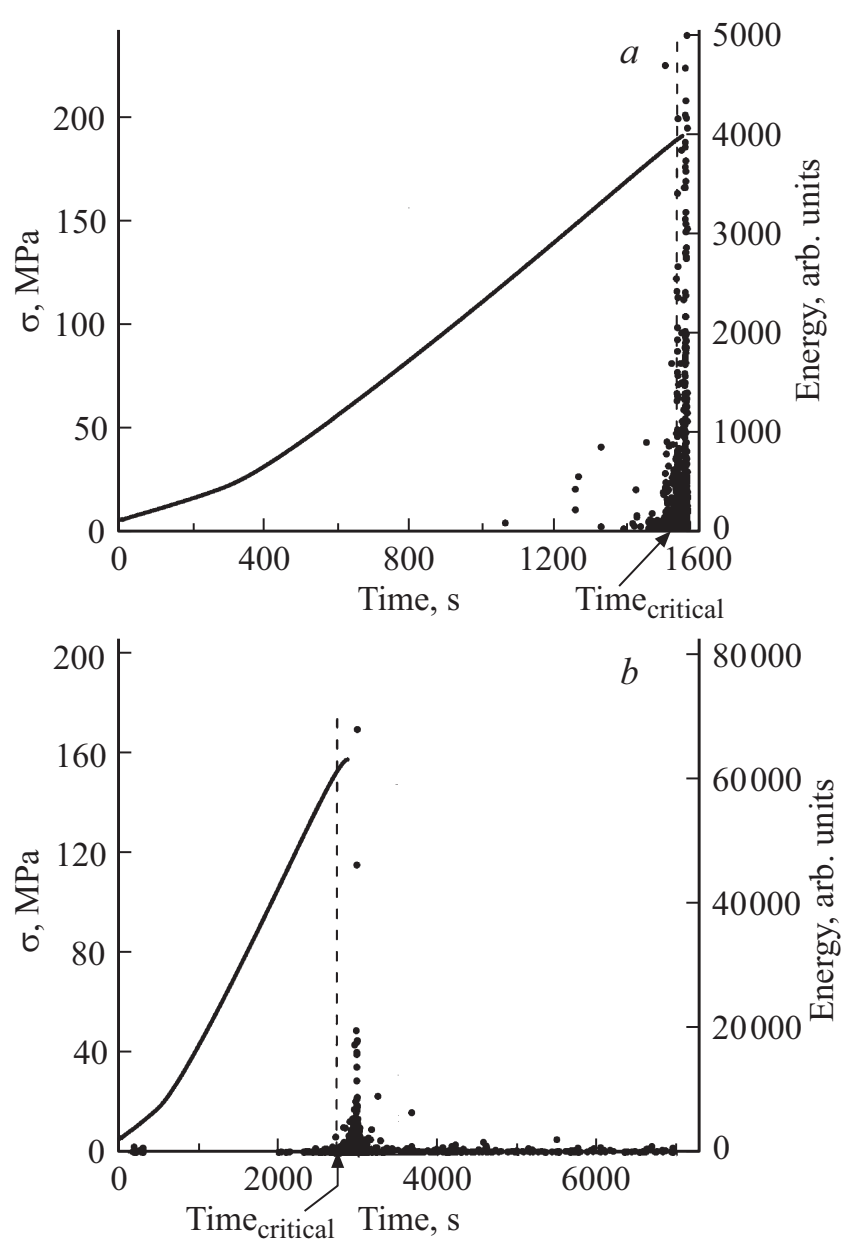

Рис. 1. Изменение напряжения (сплошная линия) и энергии отдельных сигналов АЕ (точки): $a$ - образец $\mathrm{X}, b-$ образец Y. 
стороны сформировать магистральную трещину, а с другой - сохранить целостность образца.

Всего было испытано две серии образцов (по 7 в каждой). Первая серия (обозначение „образец $\mathrm{X}^{\text {“6 }}$ ) - специально изготовленные цилиндрические образцы с неплоскопараллельными торцами (непараллельность составляет $\approx 0.5^{\circ}$ ), вторая серия („образец $\mathrm{Y}^{\text {“ }}$ ) - стандартные цилиндрические образцы с плоскопараллельными торцами. Отсутствие параллельности торцов позволяет получить при одноосном квазистатическом сжатии явно выраженную сдвиговую компоненту, что, безусловно, сказывается на процессе формирования магистральной трещины. При испытании образцов второй серии снятие нагрузки проводилось незадолго до макроразрушения образца (рис. $1, b)$, после чего процесс регистрации акустической эмиссии продолжался. Такой алгоритм нагружения позволил исследовать процесс релаксации напряжений в материале с развитой дефектной подсистемой (с фактически полностью сформированной магистральной трещиной).

\section{3. Результаты и обсуждение}

Для исследования стадийности процесса формирования магистральной трещины были использованы два подхода к анализу данных акустической эмиссии, зарегистрированной в течение всего времени деформирования: мультифрактальный анализ пауз между последовательными во времени сигналами акустической эмиссии и анализ функционального вида распределения сигналов акустической эмиссии по энергии.

\section{1. Мультифрактальный анализ пауз между последовательными во времени сигналами $\mathrm{AE}$}

В [9] было отмечено, что временны́е интервалы между импульсами акустической эмиссии связаны с интенсивностью процесса накопления повреждений в материале, а в конечном счете со скоростью диссипации подводимой к образцу механической энергии. Статистические закономерности изменения длительности пауз между импульсами АЕ обладают высокой информативностью [9-11], а в совокупности с закономерностями вариации амплитуд (энергий) позволяют охарактеризовать стадийность процесса накопления повреждений в деформируемом материале $[11,12]$.

Поскольку поток пауз является заведомо нестационарным, традиционные статистические методы (спектральный анализ, метод корреляционных функций и т.д.) к нему неприменимы. Действительно, двухвременная корреляционная функция в случае нестационарного процесса не зависит от разности времен (лага), но от каждого из времен по отдельности, а спектр мощности не может быть вычислен исходя из представлений о свертке. Однако мультифрактальные методики, основанные на вейвлет-преобразовании, таким недостатком не обладают.

Ранее было неоднократно показано, что в процессе разрушения претерпевают изменения различные фрактальные характеристики. Изменение спектра сингулярностей наблюдалось [13] при изучении трансформации рельефа латеральной поверхности аморфных сплавов под действием механической нагрузки. Для гранитных образцов было обнаружено уменьшение корреляционной фрактальной размерности ( $D$-value) множества точек, соответствующих образующимся или растущим трещинам при приближении момента разрушения $[14,15]$. В настоящей работе рассчитывались временны́е зависимости параметров спектра сингулярностей $D(h)$.

Для расчета спектра сингулярностей использовался метод вейвлет-лидеров [16,17]. Вейвлет-лидер $L(j, k)$ представляет собой наибольший коэффициент дискретного вейвлет-преобразования $d\left(j^{\prime}, k^{\prime}\right)$, вычисленный в узком временно́м интервале $(k-1) \cdot 2^{j} \leq k^{\prime} \cdot 2^{j^{\prime}} \leq$ $\leq(k+1) \cdot 2^{j}$ на всех масштабах меньших заданного $2^{j^{\prime}} \leq 2^{j}$. Вейвлет-лидеры отражают локальные коэффициенты Гельдера, которые можно рассчитать стандартным для мультифрактального анализа способом: построить обобщенную функцию распределения вида $Z(i, q) \propto \sum_{k} L(j, k)^{q} \propto 2^{j \cdot \tau(q)}$, найти из нее скейлинговые экспоненты $\tau(q)$, из которых рассчитать спектр сингулярностей $D(h)$ при помощи преобразования Лежандра. Таким образом, метод вейвлет-лидеров можно считать дальнейшим развитием метода максимума модуля вейвлет-преобразования, разработанного в [18].

Для мультифрактального анализа пауз между последовательными во времени сигналами АЕ были выбраны ряды акустических событий для двух испытанных образцов, наиболее представительных в своих сериях (образец Х и образец Y). Эти ряды состояли из 1441 и 7815 событий соответственно. Из исходных данных рассчитывались ряды пауз между ближайшими событиями. Эти ряды пауз разбивались на частично перекрывающиеся последовательности из 1024 элементов, для которых и проводился мультифрактальный анализ методом вейвлет-лидеров. Последовательности сдвигались относительно предыдущих на 16 событий для получения более гладких зависимостей от времени. Для каждой из последовательностей рассчитывался спектр сингулярностей $D(h)$. Два основных его параметра $H=h\left(D_{\max }\right)-$ (соответствует глобальному коэффициенту Херста) и ширина спектра $w$ построены на рис. 2 как функции времени деформирования.

На рис. 2, а приведены временны́е зависимости параметров спектра сингулярности для образца X (с непараллельными торцами). В этом случае наблюдается более или менее монотонное увеличение во времени коэффициента $H$ и уменьшение ширины спектра $w$. Такое поведение отражает фрактальную самоорганизацию процесса - тенденцию к монофрактализации с небольшим 

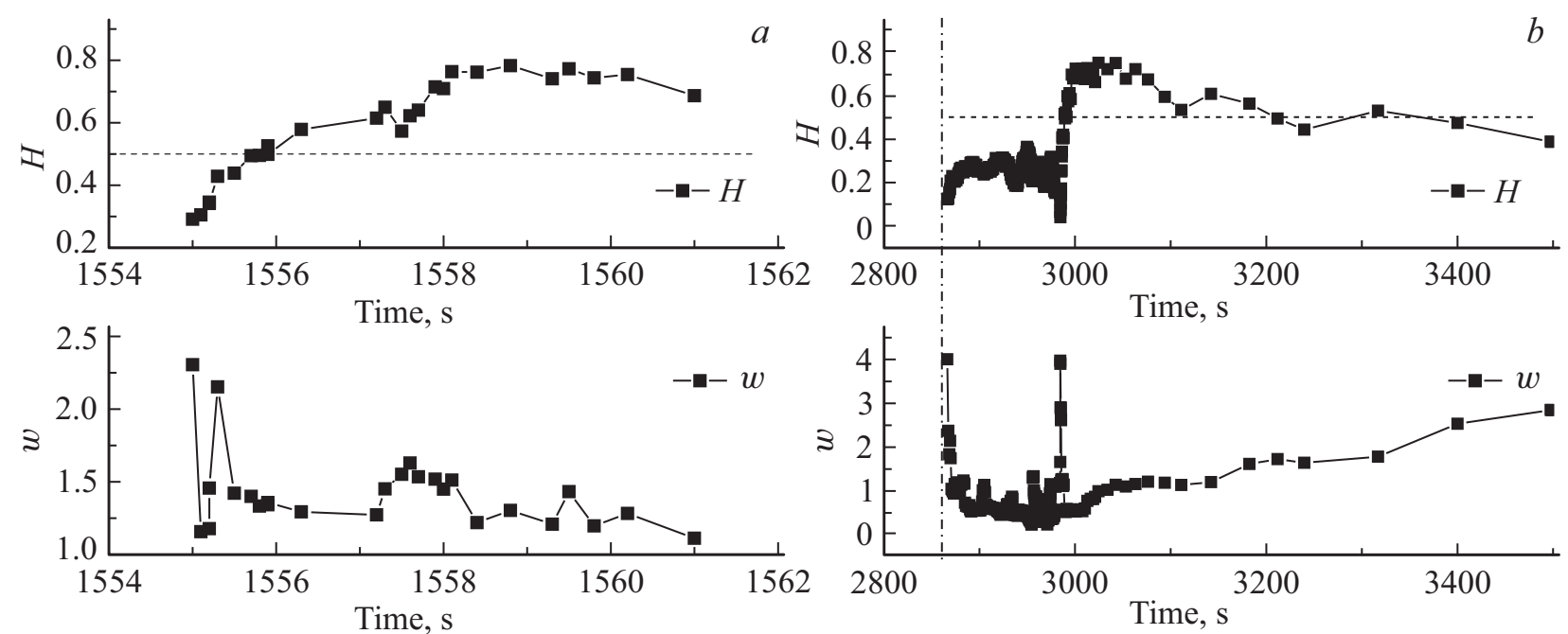

Рис. 2. Временнб́е зависимости глобального коэффициента Херста и ширины спектра сингулярностей: $a$ ) для образца Х, $b$ ) для образца Ү. Горизонтальная штриховая прямая соответствует $H=0.5$, вертикальная штрихпунктирная прямая отвечает моменту снятия механической нагрузки.

значением фрактальной размерности $D=2-H$, которая, как правило, наблюдается в процессе разрушения объема материала $[9,14]$. Заметим лишь, что в данном случае эта самоорганизация выявлена не на основе результатов пространственной локации акустических событий, а из временны́х рядов пауз между событиями. Поэтому фрактальная размерность в этом случае $D \leq 2$.

Совершенно иное поведение мультифрактальных характеристик наблюдается для образца с оставновленным нагружением незадолго до разрушения и последующей разгрузкой (рис. $2, b)$. На начальной стадии также видны тенденции к увеличению $H$ и уменьшению $w$, но далее эти тенденции пропадают вплоть до всплеска в момент времени $t \approx 2992 \mathrm{~s}$. Следует иметь ввиду, что механическая нагрузка была снята в момент времени $t=2861 \mathrm{~s}$ (отмеченный на рисунке штрихпунктирной линией), и далее процесс акустической эмиссии развивался за счет релаксации внутренних напряжений. Скачок $H$ при $t=2992 \mathrm{~s}$ можно объяснить тем, что примерно в это время и происходит рост трещины, которая наблюдается на срезах, полученных при томографии. Далее процесс некоторое время проходит в персистентной фазе $H>0.5$, после чего принимает броуновский характер, и, наконец, начинает затухать, т.е. и в этом случае развитие магистральной трещины сопровождается изменением характера процесса от более сложного мультифрактального к более простому - монофрактальному, т.е. фрактальной самоорганизацией.

\section{2. Анализ энергетических распределений сигналов $A E$}

Ранее в работах [19-22] было показано, что функциональный вид энергетического распределения сигна- лов $\mathrm{AE}$ может служить критерием состояния деформируемого материала. Установлено, что накоплению дефектов, распределенных случайным образом по объему, соответствует экспоненциальное распределение. При переходе к локализованному дефектообразованию вид распределения изменяется на степенной.

В данной работе был проведен детальный анализ функционального вида распределений сигналов $\mathrm{AE}$ по энергии. Во всех экспериментах с помощью метода последовательных приближений удалось определить момент времени Time тическое распределение становится степенным.

На рис. 3 показано энергетическое распределение сигналов AE, зарегистрированных до момента Time critical. $_{\text {. }}$ Распределение построено в двойных логарифмических (рис. $3, a$ ) и в полулогарифмических (рис. $3, b$ ) координатах. Видно, что в обоих случаях данные аппроксимируются прямой с практически равным коэффициентом детерминации $R^{2}$. Это означает, что данное распределение может быть аппроксимировано как экспоненциальной, так и степенной функцией. Подобный результат был получен нами и при анализе других экспериментов по деформированию образцов гранита [21]. Согласно гипотезе, выдвинутой в [20], такая картина означает, что материал образца находится в неопасном состоянии.

На рис. 4 показано энергетическое распределение сиг-

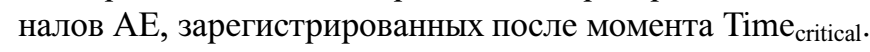
Видно, что это распределение аппроксимируется степенной функцией. Данный вид функции распределения указывает на то, что система дефектов в образце перешла в состояние самоорганизованной критичности [23]. А это, в свою очередь, означает, что материал находится в критическом состоянии, которое может привести к катастрофическому разрушению в виде потери целостности образца. 

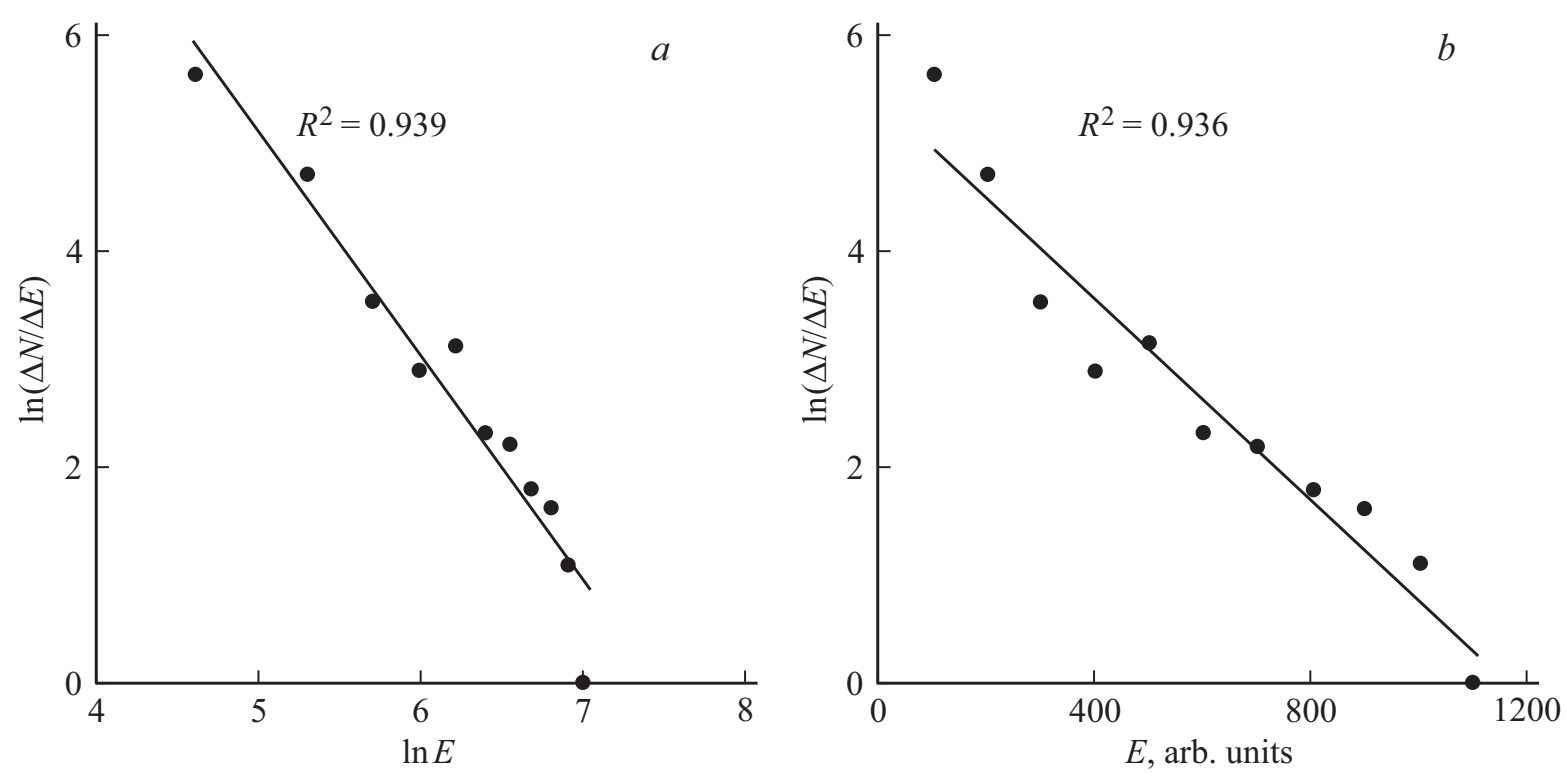

Рис. 3. Распределение по энергии сигналов AE, зарегистрированных до момента времени Time critical.
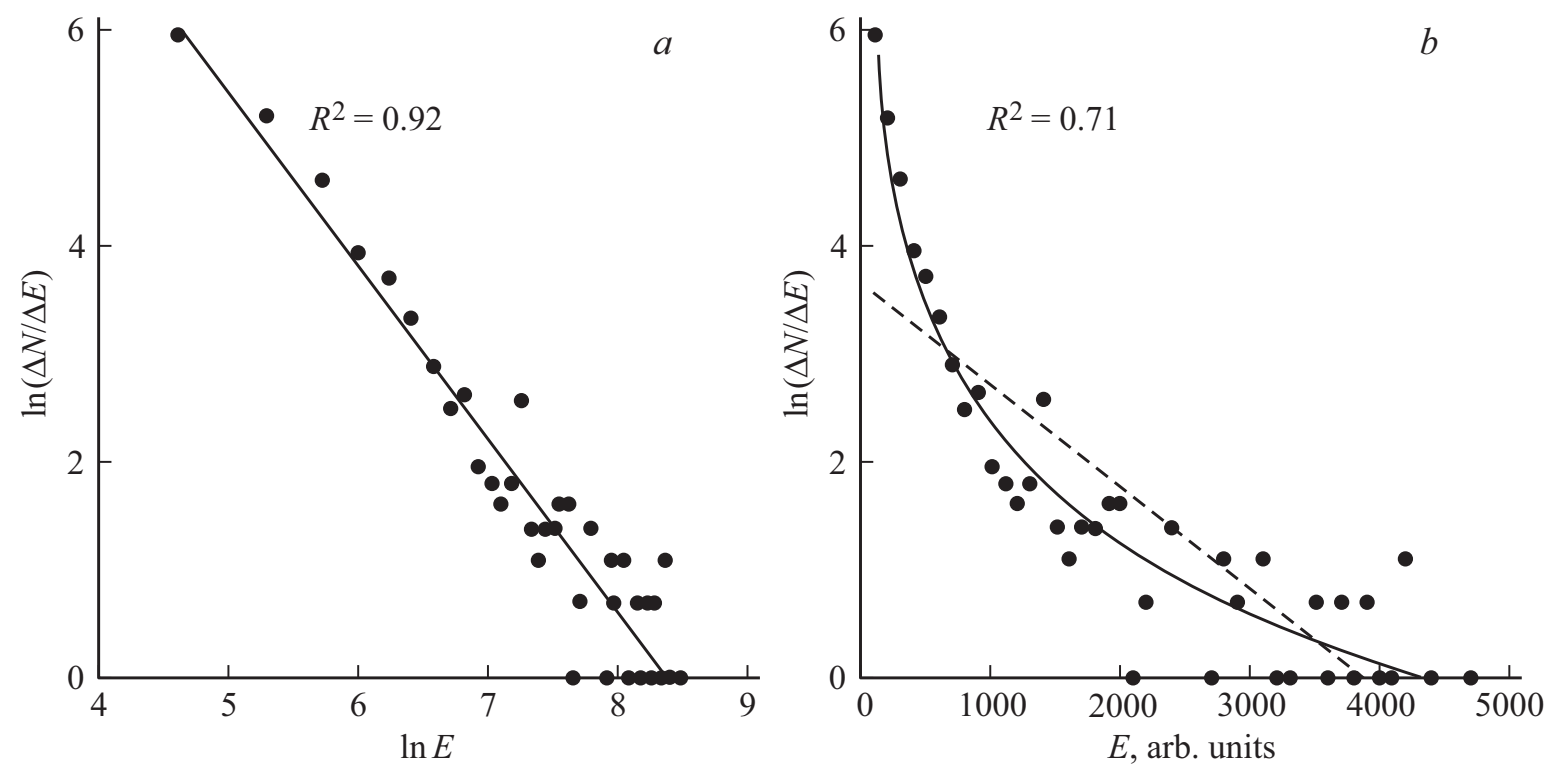

Рис. 4. Распределение по энергии сигналов АЕ, зарегистрированных после момента времени Time

Однако в экспериментах, как уже отмечалось, образцы сохранили целостность, что позволило провести томографическую съемку. На основе анализа томографических срезов, проведенного с помощью специальных программных пакетов CTan и CTvol, были построены трехмерные модели дефектной структуры (рис. 5). Видно, что в образце $\mathrm{X}$ (рис. $5, a$ ) образовалась одна плоская трещина, параллельная оси образца, расположенная ближе к его краю, что вызвано отсутствием параллельности торцов образца и указывает на преимущественно сдвиговой характер ее формирования.

Трехмерная область локализации микротрещин (магистральная трещина) в образце Y имеет более сложную форму (рис. $5, b)$ и наклонена относительно оси деформирования на некоторый угол, что является типичным для одноосно деформируемых образцов с трением на торцах. Важно отметить, что в обоих образцах все дефекты образовались только в области магистральной трещины, в других частях образца дефектов не обнаружено. Следовательно, в этих экспериментах не наблюдалось дисперсного накопления дефектов вне области трещины.

В работах [19-21] нами было установлено, что функциональный вид энергетического распределения сигналов $\mathrm{AE}$ может использоваться как индикатор текущего состояния деформированного материала. Экспоненци- 


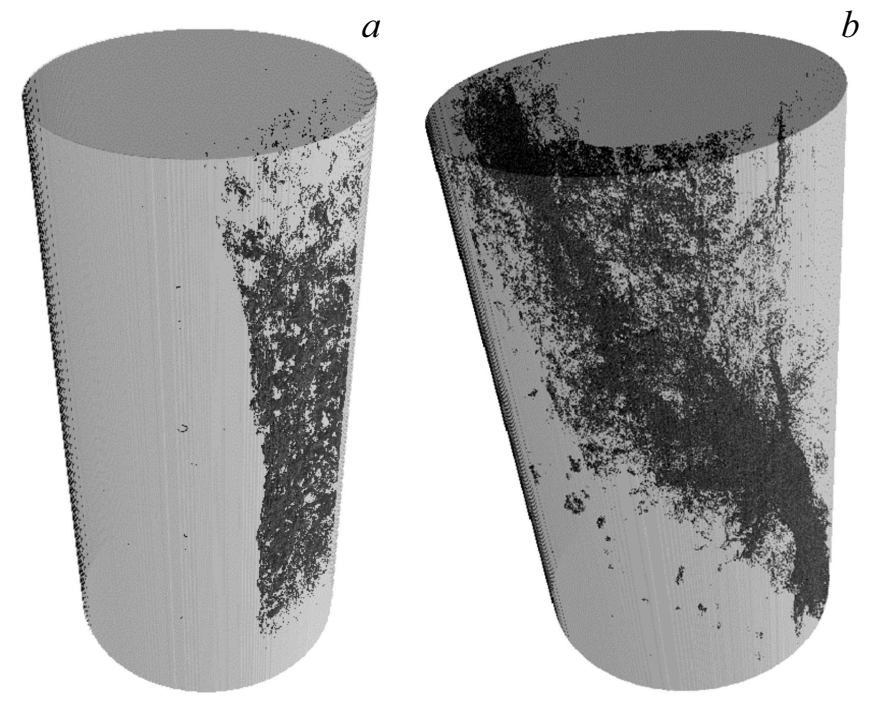

Рис. 5. Трехмерные модели дефектной структуры, построенные по данным рентгеновской томографии: $a$ - образец $\mathrm{X}$, $b$ - образец Y.

альный вид энергетического распределения сигналов АЕ указывает на некритическое (стабильное) состояние деформированного материала. Степенной вид распределения указывает на то, что процесс накопления дефектов перешел на критическую („опасную“) стадию.

В данной работе, несмотря на локализованный (в пространстве) характер накопления дефектов, выявленный методом рентгеновской микротомографии, анализ энергетических распределений сигналов акустической эмиссии позволил выделить принципиально различные стадии развития магистральной трещины. Это свидетельствует об универсальности используемого подхода и найденных закономерностях.

\section{4. Заключение}

В работе обнаружены особенности накопления дефектов при деформировании образцов в условиях квазистатического сжатия без бокового подпора. Данные компьютерной томографии показывают, что дефекты образуются только в области будущей магистральной трещины, т.е. стадии диспресного накопления дефектов во всем объеме образца не выявлено. Установлены (обнаружены) две стадии формирования магистральной трещины, первая характеризуется распределением сигналов АЕ по энергиям, которое можно аппроксимировать как экспоненциальным, так и степенным законом, вторая характеризуется степенным законом распределения сигналов AE по энергиям. Данный результат позволяет говорить о том, что формирование магистральной трещины также является процессом многостадийным, с присущей таким явлениям сменой стадии равновесной устойчивой эволюции на стадию неустойчивого развития. Подтверждением этого заключения являются результаты мультифрактального анализа пауз между соседними сигналами АЕ, согласно которому в обоих сериях наблюдается переход от мультифрактальной динамики акустической эмиссии к монофрактальной при приближении к моменту разрушения.

Авторы выражают благодарность М.Ю. Пузанкову за проведение петрографических исследований материалов образцов.

\section{Список литературы}

[1] D.A. Lockner, J.D. Byerlee, V. Kuksenko, A. Ponomarev, A. Sidorin. In: Fault Mechanics and Transport Properties of Rocks / Eds B. Evansand, T.-F. Wong. L. Academic Press. (1992). P. 3.

[2] Y. Ben-Zion, V. Lyakhovsky. Pure Appl. Geophys. 159, 2385 (2002).

[3] Y. Hamie, O. Katz, V. Lyakhovsky, Z. Reches, Yu. Fialko. Geophys. J. Int. 167, 1005 (2006).

[4] T.H.W. Goebel, T.W. Becker, D. Schorlemmer, S. Stanchits, C. Sammis, E. Rybacki, G. Dresen. J. Geophys. Res. 117, B03310 (2012).

[5] M. Petružálek, J. Vilhelm, V. Rudajev, T. Lokajíček, T. Svitek. Int. J. Rock Mech. Mining Sci. 60, 208 (2013).

[6] T. Tóth, R. Hudák. Acta Mech. Slovaca 17, 4, 40 (2013).

[7] F. Chayes. Am. J. Sci. 248, 378 (1950).

[8] R.M. Stesky. Can. J. Earth. Sci. 15, 361 (1978).

[9] И.А. Пантелеев, Ю.В. Баяндин, О.Б. Наймарк. Физ. мезомеханика 19, 4, 64 (2016).

[10] Н.Г. Томилин, Е.Е. Дамаскинская, П.И. Павлов. Физика Земли 8, 69 (2005).

[11] А.З. Красильников. Статистическая кинетика делокализованного разрушения. Автореф. канд. дис. (1991). 18 с.

[12] С.И. Буйло. Дефектоскопия 8, 79 (2004).

[13] В.Л. Гиляров, В.Е. Корсуков, П.Н. Бутенко, В.Н. Светлов. ФTT 46, 1806 (2004).

[14] В.Л. Гиляров. ФТТ 36, 2247 (1994).

[15] В.Б. Смирнов, А.В. Пономарев, А.Д. Завьялов. Физика Земли 1, 38 (1995).

[16] H. Wendt, P. Abry, S. Jaffard. IEEE Signal Proc. 24, 38 (2007).

[17] H. Wendt, S.G. Roux, S. Jaffard, P. Abry. Signal Proc. 89, 1100 (2009).

[18] J.F. Muzy, E. Bacry, A. Arneodo. Phys. Rev. E 47, 875 (1993).

[19] E. Damaskinskaya, V. Hilarov, D. Frolov. AIP Conf. Proc. 1783, 020033 (2016); DOI: 10.1063/1.4966326.

[20] E. Damaskinskaya, D. Frolov, D. Gafurova, D. Korost, I. Panteleev. Interpretation 5, 4, SP1 (2017); DOI: https://doi.org/10.1190/int-2016-0222.1

[21] Е.Е. Дамаскинская, А.Г. Кадомцев. Физика Земли 3, 78 (2015).

[22] Л.Р. Ботвина. Физика Земли 10, 5 (2011).

[23] P. Bak. How Nature Works: the Science of Self-Organized Criticality. Springer-Verlag (1996). 212 c.

Редактор Т.Н. Василевская 